Article

\title{
Régimes de mobilité. La géographicité du droit à travers l'exemple de la loi sur la mobilité de Berlin.
}

Mobility regimes. The geographicity of law through the lens of the Berlin law on mobility.

\author{
Mathis Stock \\ Professeur de géographie du tourisme, Université de Lausanne, Institut de Géographie et \\ Durabilité
}

\section{Résumé}

Le terme de régime de mobilité est devenu important pour l'étude des régulations différentielles des mobilités et permet de répondre au problème de l'étude des relations inégales de pouvoir qui structurent les mobilités, avec des formes de mobilité encouragés, soutenus ou licites par rapport à d'autres interdits, régulés, empêchés, criminalisés (Hannam et al., 2006; Cresswell, 2006). L'article vise d'abord à placer le droit comme un chaînon manquant de la géographie théorique. La notion de «géograpicité du droit » est proposée pour mettre cette dimension au centre de la géographie. Ensuite, la régulation des mobilités est analysée à travers la notion de régime de mobilité, qui est développée en focalisant sur l'articulation régulatoire de multiples échelles et domaines. Enfin, l'exemple de la loi sur la mobilité de 2018 à Berlin est développé pour montrer comment la loi opère un «mobility turn » qui modifie radicalement la politics des mobilités et permet de nouvelles normes d'habiter.

\begin{abstract}
Mobility regimes is an important notion for the study of differential regulations of mobilities and allows for answering the question of unequal power relations that structure the different modes of mobilities, some being encouraged and others forbidden, regulated, criminalised (Hannam et al., 2006 ; Cresswell, 2006). First, the article aims at positioning law as a missing link in theoretical geography. Second, the concept of mobility regime is developed as a regulation that articulates multiple scales and domains. Finally, the example of the 2018 Berlin mobility law is mobilised in order to show how law operates a «mobility turn». The detailed analysis works with the hypothesis of a radical change in the politics of mobilities allowing for new modes of inhabiting the city.
\end{abstract}

\section{Mots-clés : Mobilité - Droit - Géographie du Droit - Régime de mobilité - Berlin}




\section{Keywords : Mobility - Law - Legal Geography - Mobility Regime - Berlin \\ Régimes de mobilité. La géographicité du droit à travers l'exemple de la loi sur la mobilité de Berlin.}

\section{Introduction}

La question des mobilités est devenue cruciale avec l'émergence de styles d'habiter polytopiques (Stock, 2006), des modes de vie transnationaux (Pries, 2008) ainsi que la démultiplication des multiples mouvements fonctionnels spécifiques tels que les trajets domicile-travail, des mouvements touristiques et de loisir, d'achalandage, de voyages d'affaires, d'obligations familiales et amicales, ainsi que les migrations internationales, nationales ou régionales. La mobilité est en même temps conditionnée depuis une vingtaine d'années par la mise en place de la digitalisation des activités - appelé « révolution numérique » ou amplification du numérique (Boullier, 2016 ; Beaude, 2008) - qui posent la question de la substitution de la télécommunication au mouvement corporel des personnes pour des activités telles que les démarches administratives, les achats, le travail, le loisir. Bien que l'étude ici se concentre sur les mobilités de personnes, le terme de «mobilité » peut délimiter un ensemble phénoménal plus large : mouvements matériels et immatériels (Urry, 2005), incluant aussi la «motilité », c'est-à-dire la disposition à, et les potentiels de la mobilité (Kaufmann, 2013) ainsi que les valeurs et imaginaires (Lussault \& Stock, 2003 ; Cresswell, 2006) et des régimes de mobilité (cf. ci-dessous). Ainsi, le terme «mobilité » pourrait comprendre les flux de personnes, de biens de consommation et d'information comme étant articulés dans un système interdépendant ${ }^{1}$. Les modes d'habiter contemporains peuvent être compris comme un système où les individus agencent coprésence, mobilité et télécommunication de façon individuelle.

Cette importance sociétale accrue du phénomène se double de l'augmentation de l'intérêt

\footnotetext{
${ }^{1}$ De ce point de vue, le travail de Zelinsky (1971) sur la transition mobilitaire et la substitution possible entre migration et circulation ainsi qu'entre circulation et communication est crucial tout comme le travail de Lévy (1994) sur les effets des différentes métriques spatiales - co-présence, mobilité, télécommunication - sur les spatialités qui va, étonnamment, dans le même sens. Cf. aussi la définition «mobilité » dans le Dictionnaire de la géographie (Lussault \& Stock, 2003 ; Kaufmann, 2013).
} 
scientifique : on parle depuis une dizaine d'années d'un « mobilities turn » (Sheller et al., 2006) qui modifie profondément l'ordre scientifique en raison de l'émergence d'un champ scientifique interdisciplinaire appelé «mobilities studies » qui intègre de multiples disciplines auparavant séparées, et synthétise de multiples savoirs restés épars (Ortar et al., 2018). La définition du concept de «mobilité » a ainsi évolué, passant d'une simple définition d'ordre démographique flux de personnes et déplacement physique - à une définition plus complexe qui intègre signification du déplacement, pratiques, le capital spatial, ainsi que la «motilité » comme déplacements virtuels et comme «champ des possibles » (Kaufmann, 2013). Du point de vue du droit, la mobilité est une « activité fondée sur des droits » (Prytherch, 2012, p.301), mais reçoit des commentaires ambiguës : pour les uns, la mobilité est un droit de liberté (Ascher, 2004) qui gagne en importance dans l'Union Européenne suite à la mise en application du traité de Lisbonne qui prévoit la «libre circulation» des personnes et des marchandises. Pour d'autres, la mobilité est aujourd'hui suspecte en raison de la crise écologique (cf. Gössling, 2019) ou de la crise migratoire et doit être plus strictement encadrée. La mobilité est donc une catégorie descriptive, mais devient aussi une catégorie normative et morale utilisée par de multiples acteurs en fonction de leurs convictions et de leurs intérêts. Le droit à la mobilité, mais aussi le droit à l'immobilité (Blomley, 1994) sont ainsi invoqués.

Le terme « régime de mobilité » a été élaboré pour étudier la régulation différentielle des mobilités à l'échelle internationale : «we deploy the term 'regimes of mobility' (...) to explore the relationships between the privileged movements of some and the co-dependent but stigmatised and forbidden movement, migration and interconnection of the poor, powerless and exploited (Glick Schiller \& Salazar, 2013, p.188). Ce terme permet donc de travailler à l'étude des relations inégales de pouvoir qui structurent les mobilités, encouragent certains modes de mobilité, les soutiennent ou à défaut les autorisent et en interdisent d'autres, les empêchent voire les criminalisent (Hannam et al., 2006 ; Cresswell, 2006). Souvent posée comme un problème de géographie politique ou comme relevant d'une dimension politique, la question du pouvoir tout comme celle d'empowerment par la mobilité peuvent être posées à travers l'approche de la legal geography. Celle-ci est en développement au sein de la géographie anglo-saxonne depuis les années 1990 (Blomley et al., 2003 ; Delaney, 2003), mais se trouve enrichie plus récemment par le spatial turn 
du droit ${ }^{2}$. Ce courant propose de prendre en compte à la fois la constitution des dimensions spatiales par le droit, mais aussi la production du droit par des contextes spatiaux spécifiques. En effet, comme le dit Blomley (1994, p.51), « legal practice serves to produce space yet, in turn, is shaped by a sociospatial context ». La mobilité est précisément une activité à base légale, variables selon les contextes légaux, qui co-produit les arrangements spatiaux : récemment, le changement des accessibilités en France avec les cars interurbains (depuis la loi n 2015-990 du 6 août 2015) en offre un exemple.

Il se pose la question dans quelle mesure les normes juridiques concernant les mobilités se sont modifiés. Comment peut-on interpréter le renouveau des législations portant sur les mobilités ? Je formule l'hypothèse que les normes juridiques se sont modifiés au cours de la période 2000-2020 en Europe. Le régime de mobilité évolue : il est à fois régulé de manière plus précises et plus ambigu en encadrant les mobilités en fonction des statuts légaux des citoyens et en régulant les modes de transport en fonction de leurs valeurs écologiques.

L'investigation de ces régimes de mobilité en pleine évolution se fera en trois temps. D'abord, j'aborde les enjeux du droit pour la géographie théorique pouvant devenir une ressource conceptuelle centrale. En effet, ce texte part de l'hypothèse que les questions de pouvoir, de justice spatiale ou d'inégalités spatiales ont été appréhendées en géographie en éludant la question du juridique . Pour pallier ce manque, la notion de « géograpicité du droit » est proposée pour mettre cette dimension au centre d'un projet de géographie théorique. Ensuite, pour comprendre l'étendue de la régulation des mobilités, la notion de régime de mobilités est développée comme l'articulation régulatoire de multiples échelles et domaines de la mobilité. Enfin, l'exemple de la récente loi sur la mobilité à Berlin de 2018 est analysé en détail pour montrer comment la loi opère un «mobilities turn » qui modifie radicalement la politics des mobilités, c'est-à-dire les relations de pouvoir asymétriques, et permet l'émergence de nouvelles normes et manières d'habiter.

\footnotetext{
${ }^{2}$ Cf. par exemple les contributions de Brighenti (2006), Philippopoulos-Mihalopoulos (2011), Valverde (2005), Zick (2006), Erbsen (2011), Siehr (2016) ou encore Franz von Benda-Beckmann, Keebet Benda-Beckmann \& Anne Griffiths (2009), Caillosse (1995).

3 Cf. la revue scientifique Justice spatiale/Spatial justice (jssj.org), et les contributions de Pirie (1983), Soja (2010) et Lévy et al. (2018) pour le développement du champ de recherche de la justice spatiale en géographie.
} 


\section{L’enjeu cognitif du droit comme élément de la théorie géographique}

Il existe depuis les années 1990 une «géographie du droit » (legal geography) dans le monde anglophone, plus rarement dans le monde germanophone et francophone. Le développement d'une « legal geography » date des efforts de Blomley (1994) et Delaney $(2003 ; 2015 ; 2106$; 2017) qui ont proposé une prise en compte de textes juridiques pour comprendre la manière dont l'espace et la circulation sont organisés ${ }^{4}$. Il s'agit d'analyses qui renouvellent la compréhension de l'espace public, de la marche pédestre, mais aussi de la justice environnementale. A la fois l'analyse des effets du droit sur la constitution des espaces («law in space ») et l'analyse du contenu du droit en termes de catégories spatiales ( «space in law ») est proposée. Mélé (2009) remarque que « le droit n'est pas absent de la géographie française, il est simplement considéré comme une réalité extérieure aux espaces ou aux processus étudiés. Une vision positiviste du droit semble dominer : celui-ci est, le plus souvent, interprété comme un instrument au service d'une volonté politique proposant une prise en compte institutionnelle des problèmes sociaux » (p.26). Certes, on peut argumenter en faveur de la disparition du droit derrière l'action publique ou les politiques publiques, voire critiquer une vision positiviste du droit comme réalité indépendante des pratiques, symboliques et matérialités, comme norme pure. Néanmoins, cette assertion élude le fait que le droit concerne aussi bien le droit privé que le droit public, le droit international que le droit des affaires, le droit de la famille que le droit commercial, le droit constitutionnel que le droit environnemental. Ces domaines ne sont ni analysés comme «extérieur aux espaces et aux processus étudiés » selon la citation de Mélé (2009) ni quant à leurs référents géographiques ou « géographicités », mais simplement ignorés ${ }^{5}$. Les seuls domaines où des notions de droit sont considérées, concernent l'étude de l'urbanisme règlementaire dans les analyses de l'aménagement ou la régulation urbaine dont l'action normative et légale contribue à la fabrique de l'espace (Bourdin et al., 2005).

\footnotetext{
4 On pourrait retracer jusque dans les années 1920 les premières tentatives de développer une « géographie du droit » dans une perspective culturaliste et positiviste, ainsi que la critical legal geography depuis les années 1990 et le récent tournant spatial du droit, mais cela n'est pas possible ici faute de place. Les questions foncières ont nécessité de la part des géographes une compréhension des enjeux juridiques, mais sans nécessairement aller jusqu'à intégrer les dimensions juridiques dans un cadre théorique cohérent.

${ }^{5}$ Exceptions : Mélé (2009) sur la régulation urbaine et l'ordre local du droit, Garcier $(2009$; 2014) pour le droit environnemental et Maccaglia (2009) sur les illégalismes à Palerme. Cf. Forest (2009) pour un volume sur la géographie du droit.
} 
Il y a un enjeu cognitif à développer le droit comme un élément d'une géographie théorique. Par « géographie théorique »,j'entends l'ensemble des pratiques de formalisation, de conceptualisation et de modélisation en géographie. Notamment, l'articulation des dimensions spatiales aux théories sociales, y compris celles du droit, constitue l'horizon contemporain de la géographie. L'enjeu réside dans la capacité à préciser la question du pouvoir et des normes, maintenant classiques en géographie, en faisant intervenir dans les analyses également le droit formel. L'enjeu réside aussi dans la capacité à analyser les sources d'empowerment ou de pertes de pouvoir - typiques de la mobilisation d'un concept relationnel de pouvoir (Elias, Foucault) - en identifiant le droit comme l'un des éléments possibles. Ainsi, il s'agit de proposer l'intégration des dimensions juridiques comme outil théorique dans les cadres d'analyse en géographie.

Comment pourrait-on intégrer les multiples dimensions du droit dans la géographie théorique ? La géographie contemporaine développant de perspectives théoriques multiples, les dimensions juridiques peuvent être développées dans chacune d'elles, que ce soit en géographie économique, politique, culturelle, sociale. La géographicité du droit peut être analysée quelles que soient les thématiques : justice spatiale, développement économique, pratiques spatiales, habiter, mobilités, urbanisation, urbanité, etc.. Ainsi, il semble que deux voies peuvent être poursuivies : 1) la prise en compte de la dimension juridique dans les modèles multiples de la géographie; 2) le développement par la notion de «géographicité du droit » pour analyser la production du droit d'une part et la production de l'espace d'autre part.

\subsection{Les dimensions juridiques dans les théories de la pratique}

Depuis le «practice turn » en géographie, les modèles d'action cadrent les investigations en direction de la spatialité des pratiques, que celles-ci soient individuelles ou institutionnelles. En effet, la théorisation en géographie a massivement porté, depuis les années 1980, sur les modèles d'action qui pointent les éléments suivants : des actions porteuses de sens (encodage/décodage selon Alfred Schütz), mobilisant des ressources (capital social, économique, culturelle et symbolique selon Pierre Bourdieu), subissant des contraintes ou des dominations (Elias, Bourdieu), permettant des marges de manœuvre (agency selon Anthony Giddens ou tactiques et résistances 
selon Michel De Certeau), engageant des identités plurielles (Lahire, 1998) dans des modes de vie extrêmement différenciés ${ }^{6}$.

Ces pratiques sont normées par des normes informelles, mais aussi formelles, c'est-à-dire juridiques. Le droit confère aussi des ressources dans le sens d'empowerment et d'agency des individus. En effet, les processus d'augmentation ou de restriction des libertés individuelles se passent aussi, dans un Etat de droit, par les dispositifs légaux. Le droit serait ainsi une pratique qui engage de multiples actants dans des discours de type normatif. Ce qui implique aussi de «faire avec le droit » au sens où la pratique du droit correspond à une pratique négociée : «même au sein de l'univers par excellence de la règle et du règlement, le jeu avec la règle fait partie de la règle du jeu » (Bourdieu, 1990, 89). Ainsi, les pratiques spatiales sont confrontées aux normes juridiques où le droit comme normativité est activé.

La prise en compte du droit comme moyen d'action à la place de la violence physique, des moyens financiers ou du capital culturel, social, économique paraît jusqu'ici sous-développée dans les cadres théoriques. L'un des enjeux théoriques semble être d'appréhender le droit comme source d'empowerment et comme source de perte de pouvoir dans les stratégies spatiales des individus et opérateurs collectifs. C'est ainsi que le droit peut être intégré dans les théories de l'action ou de la pratique comme norme formelle dans un ensemble pratique.

\subsection{L'étude de la "géographicité du droit » comme apport théorique?}

Le concept de «géographicité du droit » peut être développé. Il désigne cette double dimension d'une étude des dimensions juridiques de la constitution d'agencements et dispositifs spatiaux et des dimensions géographiques des ordres juridiques pluriels. Par «géographicité » du droit, j'entends à la fois l'étude de la constitution légale de l'espace (agencement, accessibilité, limites, localisation, aires, etc.), mais aussi la manière dont les différentes dimensions spatiales sont imaginées et traduites dans des textes de loi par le truchement de catégories spatiales. Ainsi, le

\footnotetext{
${ }^{6}$ Cf. le travail de Thrift (1996), Werlen $(1993$; 1997), Lussault (2007) pour la théorisation géographique de ces éléments, et Stock (2015) concernant l'utilisation de ces éléments dans une théorie de l'habiter.
} 
droit peut se loger dans l'ensemble de problèmes posés par la géographicité des sociétés humaines, allant de la définition des lieux géographiques à la production d'espaces sociaux spécifiques en passant par les questions de spatialités quelles que soient leur expression (rapport à la distance, à la limite, à la localisation, etc.).

Le terme de «géographicité » a été développé dans la géographie francophone pour désigner d'abord la géographie phénoménologique et existentialiste : « (...) Connaître l'inconnu, atteindre l'inaccessible, l'inquiétude géographique précède et porte la science objective. Amour du sol natal ou recherche du dépaysement, une relation concrète se noue entre l'homme et la Terre, une géographicité de l'homme comme mode de son existence et de son destin » (Dardel, 1952, p. 2, souligné par moi). Cette dimension ontologique est centrale, notamment pour Berque (1999) qui nomme « géographicité » la dimension géographique des humains en tant qu'ils vivent sur Terre. Retaillé (2003, p.398) définit dans le Dictionnaire de la géographie et de l'espace des sociétés la géographicité comme «la relation ontologique et phénoménologique existant entre l'homme et l'espace ». Sur un plan différent, Raffestin (1989) plaide « pour une théorie de la géographicité » (1989, p.29), terme qui y désigne la pratique et la connaissance de la Terre, non la Terre en tant que telle.

Le terme « géographicité » est construit ici par analogie avec le terme « historicité » pour signifier les multiples dimensions spatiales et référents géographiques qui sont impliqués dans l'ensemble des problèmes des sociétés humaines, comme « régime de géographicité » (Stock, 2003-2004)? Ainsi compris, on peut appréhender la «géographicité des normes ». Selon Stock $(2015,432)$, « utilisé dans l'analyse de l'habiter, ceci signifie une attention à la géographicité des normes qui est constitutive des pratiques. Ainsi, exécuter des normes spatiales (par exemple en termes de distance entre corps, de limites posées, etc.), qu'elles soient formelles ou non formelles, énoncées ou non, relève de la pratique, car ces normes spatiales sont toujours négociées ». Ainsi, la géographicité du droit serait un élément spécifique en ce qu'il s'agit de normes formelles. La géographicité du droit relève alors de quatre éléments :

\footnotetext{
${ }^{7}$ En cela, il pourrait dialoguer avec la définition modifiée du Dictionnaire de l'espace et des sociétés de 2013 : la géographicité comme « composante spatiale de la sociétalité. Elle réunit la spatialité comme agir spatial et l'espace comme environnement de cet agir » (Lévy, 2013, p.434).
} 
1. Le droit comme un ordre discursif de l'espace, un ordonnancement discursif de l'espace. Le géographe peut alors étudier les textes de lois, de jurisprudence ou de dogmatique dans lesquels les catégories spatiales sont utilisées. Les multiples référents géographiques - distances, limites, localisations, qualités de lieu, toponymes, aires, réseaux, etc. - qui apparaissent dans les textes juridiques peuvent être analysés.

2. Le droit comme ordre normatif, car il prescrit comment les individus devraient agir avec l'espace et qui produit différentes qualités de lieu, des distances, de limites etc.. En cela, il devient norme spatiale ; le jeu avec la norme spatiale fait également partie de la géographicité du droit. 3. Les dispositions juridiques concernent les objets traditionnels de la géographie tels que la nomenclature et la toponymie des lieux géographiques. Ainsi, du point de vue épistémologique, la codification juridique dédouble les objets géographiques en leur assignant une existence juridique. 4. Les ordres juridiques localisés à différents niveaux d'échelle, produits par différents acteurs autour de conflits et controverses. Ceci prend notamment sens avec la mondialisation du droit dans le sens d'une complexité normative grandissante.

\subsection{Conclusion}

Cette contextualisation de la prise en charge des questions juridiques par la géographie semble nécessaire pour indiquer différents usages possibles du droit. Allant de la simple utilisation pour élucider des études de cas à l'intégration dans une géographie théorique, en passant par le développement de notions spécifiques, plusieurs options sont possibles. La géographie contemporaine étant plurielle avec de multiples emprunts théoriques, les questions juridiques peuvent prendre place dans chacune d'elles. La proposition ici consiste à intégrer systématiquement la géographicité du droit dans ses cadrages et interprétations des spatialités contemporaines. La notion de «régime de mobilité », développée dans la partie 2 comme l'ensemble des régulations des mobilités, vise à focaliser sur l'un des aspects.

\section{La notion de régime de mobilité comme élément central d'une approche légale des mobilités}


La mobilité est dorénavant intégrée dans les modes d'habiter - à bien des égards, habiter c'est être mobile - et ainsi dépendante des régimes juridiques. Le terme des «régimes de mobilité » appréhende les modes de régulation des mobilités, car celles-ci sont inégalement encouragées ou au contraire interdites ou contrôlées par des dispositifs légaux. Cresswell (2006, p. 735) pose la question de savoir « how particular modes of mobility are enabled, given licence, encouraged and facilitated while others are, conversely, forbidden, regulated, policed and prevented ». C'est en effet la question centrale dont la réponse varie en fonction des formations sociales. Les régimes de mobilité posent aussi la question des relations de pouvoir et des inégalités en termes d'accessibilité et de mouvements : il existe en effet des « unequal power relations which unevenly distribute motility, the potential for mobility » (Hannam et al., 2006, p. 15). Ces différentiels de motilité sont, entre autres, fondés sur une distribution des droits : la mobilité est elle-même une activité ou un ensemble d'activité fondés sur des droits inégaux et des contraintes légales.

L'expression de «régime de mobilité » a été mis en place par Shamir (2005) concernant les migrations dont l'accès aux lieux étatiques est régulé : «We are witnessing the emergence of a global mobility regime, oriented to closure and to the blocking of access, premised not only on "old" national or local grounds but on a principle of perceived universal dangerous personhoods » (Shamir, 2005, p.199). Il montre que ce régime de mobilité repose sur des technologies spatiales (frontières, camps, ghettos) et un profilage bio-social qui différencie les individus selon leur utilité sur un marché d'emploi et selon le niveau de menace dont ils sont porteurs. Ainsi, au lieu de concevoir le régime de mobilité comme ouvert, Shamir (2005) conçoit un régime de mobilité très finement organisé qui réduit l'accès à certains droits. Il est mondial, car opère selon la même logique à l'échelle mondiale et forme ainsi un exemple de la «mondialisation du droit » (DelmasMarty, 2004). Le concept sert à analyser les relations entre des mouvements qualifiés de privilégiés et d'autres, qualifiés de stigmatisés qui sont interdits, criminalisés ou empêchés. Dans le même temps, il vise les éléments d'ordre juridique et politique de la régulation des mobilités.

Jusqu'ici, le terme « mobility régime » a été appliqué à la mobilité transnationale pour montrer les différents statuts juridiques des personnes mobiles (touristes, migrants, réfugiés, frontaliers, etc.).

\footnotetext{
8 De multiples études s'y sont engouffrées que je ne pourrais citer qu'en passant, par manque de place. Cf. notamment Koslowski (2011) et Glick Schiller \& Salazar (2013) pour l'idée de l'interdépendance des régimes de mobilité.
} 
A partir de là, on peut aller plus loin et élargir ce terme aux les dispositifs normatifs de toutes les formes de mobilité. D'abord, on peut détecter ces régimes de mobilité à différents niveaux d'échelle et pour toutes sortes de mobilités, pas seulement pour les mobilités transnationales. Ensuite, la régulation ne porte pas seulement sur les statuts juridiques des citoyens mobiles, mais aussi sur un marché (le secteur des transports) et des systèmes techniques. Enfin, la mobilité met en relation les personnes avec certaines qualités d'espace : la traversée d'espaces privés et d'espaces publics ainsi que la capacités physiques et techniques du corps sont à prendre en compte. Ainsi, le terme « régime de mobilité » concerne à la fois les pratiques de mouvements elles-mêmes, aux accessibilités, aux systèmes techniques, au corps, au marché économique. Cette perspective trouve sa justification dans les récentes élaborations de la mobilité comme «système » (Urry, 2005) qui comprend les corps humains, les pratiques, les technologies, le marché, les normes, etc. De cette perspective découle le cadre d'analyse suivant pour appréhender la multiplicité des régulations de la mobilité dans un régime de mobilité, en fonction du niveau d'échelle de l'ordre juridique concerné (tableau 1). Il intègre quelques exemples et domaines de régulation des multiples dimensions de la mobilité.

Tableau 1 : Les différentes dimensions du régime des mobilités et quelques exemples à plusieurs niveaux d'échelle

\begin{tabular}{|c|c|c|c|c|c|}
\hline & Statut légal de la personne & Corps physique & $\begin{array}{l}\text { Système } \\
\text { technique }\end{array}$ & $\begin{array}{l}\text { Marché } \\
\text { économique }\end{array}$ & Espace \\
\hline $\begin{array}{l}\text { Ordre } \\
\text { juridique } \\
\text { local }\end{array}$ & $\begin{array}{l}\text { Lois locales sur: } \\
\text { - Résident/non-résident } \\
\text { («Touriste » avec taxe de } \\
\text { séjour, fiche police, } \\
\text { statistique) } \\
\text { - Citoyen/étranger (Droit } \\
\text { électoral) }\end{array}$ & $\begin{array}{l}\text { Mobilité } \\
\text { («accessibilité »; } \\
\text { «barrières ») } \\
\text { Sportifs }\end{array}$ & $\begin{array}{l}\text { Règlements } \\
\text { locaux des } \\
\text { moyens de } \\
\text { transport } \\
\text { (trottinettes } \\
\text { électriques, } \\
\text { moteurs diesel, } \\
\text { etc.) }\end{array}$ & $\begin{array}{l}\text { Règlements } \\
\text { locaux sur taxi, } \\
\text { VTC, transports } \\
\text { publics, }\end{array}$ & $\begin{array}{l}\text { Code de la rue } \\
\text { (Belgique) } \\
\text { Règlements sur les } \\
\text { restrictions de } \\
\text { l'espace public } \\
\text { Règlements vitesse } \\
\text { PLU; Loi de Berlin } \\
\text { sur la mobilité } \\
(2018)\end{array}$ \\
\hline $\begin{array}{l}\text { Ordre } \\
\text { juridique } \\
\text { national }\end{array}$ & $\begin{array}{l}\text { Droit } \\
\text { constitutionnel (Nationalité, } \\
\text { Citoyenneté, Libre } \\
\text { circulation) } \\
\text { Contrôle migratoire (Visa, } \\
\text { ETA) } \\
\text { Identification }\end{array}$ & $\begin{array}{l}\text { Mobilité réduite } \\
\text { (Behindertengleichstellungs- } \\
\text { gesetz) } \\
\text { Sportifs } \\
\text { Système de santé }\end{array}$ & $\begin{array}{l}\text { Plateformes } \\
\text { web (Blabla } \\
\text { car, uber) } \\
\text { Vélo, trotinette, } \\
\text { roues } \\
\text { gyroscopiques }\end{array}$ & $\begin{array}{l}\text { Consommateur } \\
\text { (Droit du } \\
\text { voyageur) } \\
\text { Marchés } \\
\text { nationaux } \\
\text { France : } \\
\text { LOTI/LOMI }\end{array}$ & $\begin{array}{l}\text { Code de la route } \\
\text { Droit } \\
\text { constitutionnel : } \\
\text { Espace public (droit } \\
\text { constitutionnel) } \\
\text { France (loi littoral ; } \\
\text { loi montagne, etc.) }\end{array}$ \\
\hline
\end{tabular}




\begin{tabular}{|c|c|c|c|c|c|}
\hline & Droit d'asile & & $\begin{array}{l}\text { Automobile } \\
\text { autonome et } \\
\text { données }\end{array}$ & & $\begin{array}{l}\text { Allemagne: Bundes- } \\
\text { aturschutzgesetz; } \\
\text { Raumordnungsgesetz } \\
\text { Droit de propriété } \\
\text { pivée (Loi } \\
\text { fondamentale) } \\
\text { Carsharinggesetz } \\
\text { (CsgG) }\end{array}$ \\
\hline $\begin{array}{l}\text { Ordre } \\
\text { juridique } \\
\text { européen }\end{array}$ & $\begin{array}{l}\text { Traité de Maastricht sur la } \\
\text { citoyenneté européenne } \\
\text { Traité de Lisbonne et } \\
\text { Directive 2004/38 sur la } \\
\text { libre circulation } \\
\text { Convention de Schengen } \\
\text { sur la libre circulation }\end{array}$ & $\begin{array}{l}\text { Mobilité réduite } \\
\text { («accessibilité») } \\
\text { Sportifs } \\
\text { Charte des droits } \\
\text { fondamentaux de l'UE }\end{array}$ & $\begin{array}{l}\text { Flotte } \\
\text { automobile } \\
(120 \mathrm{~g} / \mathrm{kg} \mathrm{CO} 2 ; \\
\text { Directive UE } \\
(2008 / 50 / \mathrm{EC}) \\
<40 \mathrm{mg} \quad \mathrm{No} \text {, } \\
\text { etc.) }\end{array}$ & $\begin{array}{l}\text { Droit du } \\
\text { voyageur } \\
\text { Directive Cars } \\
\text { interurbains } \\
\text { Concurrence } \\
\text { ferroviaire } \\
\text { Directive } \\
\text { «libre } \\
\text { concurrence» }\end{array}$ & $\begin{array}{l}\text { Directive sur } \\
\text { l'environnement; } \\
\text { Droit de propriété } \\
\text { privée (Charte des } \\
\text { droits fondamentaux } \\
\text { de l'UE) }\end{array}$ \\
\hline $\begin{array}{l}\text { Ordre } \\
\text { juridique } \\
\text { mondial }\end{array}$ & $\begin{array}{l}\text { ONU : } \\
\text { - Convention de Genève sur } \\
\text { les réfugiés } \\
\text { - Charte de l'ONU sur les } \\
\text { voyages } \\
\text { OMT: code éthique du } \\
\text { tourisme }\end{array}$ & $\begin{array}{l}\text { Suivi antidopage pour } \\
\text { sportifs professionnels } \\
\text { (AMD) } \\
\text { Organisation mondiale de la } \\
\text { santé } \\
\text { Convention relative aux } \\
\text { droits des personnes } \\
\text { handicapées }\end{array}$ & IATA & GATTS & $\begin{array}{l}\text { Convention de } \\
\text { Vienne (1967) sur le } \\
\text { permis de conduire }\end{array}$ \\
\hline
\end{tabular}

En visant différentes dimensions du système de mobilité, cette grille d'analyse permet de préciser l'hypothèse du début, en pointant les différentes dimensions de ce changement de régime de mobilités depuis une vingtaine d'années : il s'agirait d'un régime contradictoire dont les régulations concernent les systèmes techniques, le design du marché, les statuts légaux des personnes, les capabilités physiques des corps, et le type d'espace. Ceci mène à un renforcement des contrôles migratoires en apparente contradiction avec une libéralisation des marchés de l'aviation (low cost) qui mène à une accessibilité accrue du lointain et une pratique touristique accrue des villes. En même temps, la ville fait l'objet d'une écologisation de la mobilité quotidienne, avec des règlements de plus en stricte des systèmes techniques (moteur diesel, CO2) et un renforcement des politiques de transports publics ainsi que l'émergence de co-voiturage, mais aussi sur la place du piéton dans la ville en agissant sur la qualité des espaces publics. 


\section{La loi sur la mobilité de Berlin 2018 : un « mobility turn » de la loi ?}

La régulation des mobilités est analysée ici à travers l'exemple de la récente loi sur les mobilités à Berlin. La loi sur la mobilité de 2018 de Berlin, entrée en vigueur le 18 juillet 2018, est intéressante de plusieurs points de vue'. D'abord, il s'agit d'une métropole qui se dote d'une loi en raison de son statut d'Etat (Land) au sein de la République fédérale d'Allemagne. Elle a donc des prérogatives légales fournies par la loi fondamentale (Grundgesetz) allemande. Ensuite, les objectifs de la loi visent à lutter contre les inégalités mobilitaires et exprime donc un ordre juridique local ou une culture juridique locale singulière, fondé sur l'articulation d'une ville touristique et des activités festives à l'espace des mobilités quotidiennes. Le contexte d'une mobilité de type métropolitain - mesurable par un degré de motorisation de 358 voitures/1000 habitants, un split modal automobile minoritaire (1/3 en 2017) - permet de contextualiser l'évolution des normes d'habiter ${ }^{10}$. La part modale du vélo est passée de 7 à $13 \%$ entre 1992 et 2013, celle de la voiture de 35 à 32\%, celle du pédestre de 27 à $29 \%$ pour l'ensemble de Berlin ; les quartiers centraux (Mitte et Friedrichshain-Kreuzberg) étant les moins motorisés avec des chiffres de respectivement $22 \%$ et $17 \%$ de l'ensemble des mobilités intra-urbaines (Senat Berlin, 2017). La touristification massive de Berlin depuis la réunification, passant de 10 à 30 millions de nuitées entre 1990 et 2019. La stratégie «Berlin 2018+» sur le tourisme durable évoque 30 millions de nuitées, 50 millions d'excursionnistes et 100 millions de déplacements de loisir intramétropolitains par an (Senat Berlin, 2018).

Enfin, la ville est elle-même issu d'une loi, celle du Grand Berlin de 1920 et constitue un exemple de la production de l'espace par le droit. Il s'agit de la loi sur la formation d'une nouvelle commune urbaine Berlin (Gesetz über die Bildung einer neuen Stadtgemeinde Berlin (Groß-Berlin-Gesetz)) du 27 avril 1920 (cf. figure 1). Aujourd'hui, inséré dans la loi d'aménagement du territoire qui régule le développement urbain comme d'un espace métropolitain organisé en «étoile »

\footnotetext{
9 Disponible en ligne : http://gesetze.berlin.de/jportal/portal/t/iki/page/bsbeprod.psml/action/portlets.jw.MainAction?p1=5\&eventSubmit_do Navigate $=$ searchInSubtreeTOC\&showdoccase $=1 \&$ doc $. h \mathrm{l}=0 \&$ doc $. \mathrm{id}=\mathrm{jlr}-$

MobGBEpP1\&doc.part=S\&toc.poskey=\#focuspoint. Je fournis dans ce texte les traductions de la loi en français ; en raison du manque de place, les citations originales en allemand ne sont pas reprises.

${ }^{10}$ Le terme « split modal » se réfère à la répartition des choix modaux en termes de moyens de transport.
} 
(Siedlungsstern) (cf. figure 2), l'action législative de Berlin se déroule dans un cadre administratif de 12 arrondissements (Bezirke) et 96 quartiers.

Fig. 1 : L'extension et la composition administrative de la ville de Berlin après la loi de 1920

Fig. 1 : The extension and administrative composition of the city of Berlin after le law of 1920

\section{FIGURE 1 ICI}

Source : Wittig (1931), cité d'après AIV Berlin (2017)

Fig. 2 : L'aire métropolitaine d'après le Landesentwicklungsplan de 2017

Fig. 2 : The spatial development of Berlin in the Landesentwicklungsplan of 2017

\section{FIGURE 2 ICI}

Source : Landesentwicklungsplan (2017)

\subsection{La mobilité comme « système » qui participe aux modes d'habiter}

Les objectifs de la loi visent à réguler l'ensemble des formes de mobilités, des modes de transports et des conditions d'accessibilité ainsi qu'à remédier aux inégalités mobilitaires. En effet, il s'agit de la «préservation et développement d'un système de transport sûr, sans obstacle, axé sur les besoins de mobilité de la ville et de sa périphérie, respectueux de la ville, de l'environnement, de la société et du climat » (préambule, souligné par moi). Ce système n’est pas une fin en soi, mais est vu « comme contribution à un mode de vie individuel et un espace de vie inclusif, ainsi que comme élément indispensable d'une région métropolitaine viable et durable » $(\S 1$, souligné par moi). 
Ainsi, le système de mobilités est appréhendé comme un auxiliaire des «modes d'habiter » qui sont fondés aujourd'hui sur la mobilité accrue (que ce soit au quotidien ou au hors-quotidien). Le but étant de garantir des «conditions de vie équivalentes » par un accès à la mobilité qui est indépendant des inégalités sociales en fonction de l'âge, du genre, du revenu, des handicaps physiques, de la situation personnelle, du pays de naissance (migrant ou non) ou de la disposition d'un moyen de transport. Cette mobilité doit être garantie sans discrimination spatiale ou temporelle : «tous les jours et à toute heure » et «dans toutes les parties de Berlin ». Ainsi, on pourrait parler d'un «mobility turn » de la loi, car il ne s'agit pas de réguler un secteur de transports, mais bel et bien la motilité et les modes de vie mobiles. La notion centrale est « système de mobilités » $(\S 1):$ «les différents modes de transport doivent être utilisés avec leurs forces spécifiques afin d'optimiser le système global en fonction des exigences du futur » (souligné par moi). Ce système est d'échelle locale, mais également ouvert aux articulations avec les mobilités nationales et internationales $(§ 13)$. Ce système des mobilités est régulé selon quatre dimensions choisies du tableau 1 - corps, marché, système technique, espace -, étant entendu que la cinquième dimension du statut légal de la personne n'est guère problématisée dans la loi berlinoise.

Marché. La loi cherche à réguler le split modal et régule ainsi un marché (du transport et du commerce de moyens de transport) d'échelle locale sans cependant intervenir dans l'ensemble des régulations qui se font à l'échelle de l'UE et de la République d'Allemagne, voire à l'échelle mondiale (Code de Vienne de 1967). En incitant l'usage du transports publics et du vélo, la loi contribue ainsi au design local du marché, et crée un ordre juridique local. La notion centrale dans la loi est «Umweltverbund» que nous traduisons ici par «ensemble des modes de transports écologiques »". Il est défini dans la loi comme " comprenant les moyens de transports marche à pied, cycliste et transports publics » $(\$ 2,13)$. Le préambule énonce clairement la visée : «La mobilité réglementée englobe les besoins particuliers de tous les groupes de mobilité, à savoir ceux des piétons et des cyclistes, des transports publics, du trafic commercial et des transports privés motorisés, tout en garantissant la primauté des modes de transports écologiques (Umweltverbund) » (souligné par moi). Ceci se trouve renforcé dans son paragraphe

\footnotetext{
11 Littéralement : « alliance environnementale »
} 
suivant : «En améliorant l'efficacité et l'attractivité des services de transport écologiques, la part de ceux-ci dans les distances parcourues doit être augmentée ».

Cette priorité se trouve exprimée à de multiples reprises en fonction des différentes thèmes abordés : qu'il s'agisse de financement ${ }^{12}$, de régulation du trafic ${ }^{13}$ ou d'aménagement de pistes cyclables $^{14}$. Deux tiers de la loi sont consacrés à deux éléments de cette mobilité écologique, le soutien aux déplacements cyclistes $(\$ \S 36-49)$ et le soutien au transports publics (\$§26-35). Par ailleurs, $\S 8$ sur protection de l'environnement et du climat fait le lien entre mobilité et milieux géographiques. C'est là l'un des éléments de l'écologisation du régime de mobilité ainsi que de la remise en cause d'un «espace-temps automobile» par le droit. De façon surprenante, le taxi comme mode de transport ainsi que les nouvelles formes de VTC (certes mentionnés au titre du partage de données) et de partage (voiture, vélo, etc.) de ce qui est convenu d'appeler la « sharing economy » ne sont évoqués qu'une seule fois et n’apparaissent pas comme prioritaires.

Espace. Les multiples dimensions spatiales apparaissent sous différentes formes : premièrement, comme requalification des places et rues : «Lors de l'aménagement nouveau et la refonte fondamentale des rues et des places, il devrait être examiné si et dans quelle mesure elles peuvent être utilisées (...) comme lieu de rencontre, de détente, de loisirs, de communication et de jeu » $(\S 4,5)$. Deuxièmement, mobilité et qualité des lieux géographiques sont articulés de telle façon que les projets d'aménagement sont soumis à une évaluation en termes de transports et que la mixité fonctionnelle réduit les déplacements $(\$ 7)$. Selon $\$ 7,1$, la proximité entre les différents champs d'activité est l'un des objectifs de l'aménagement pour éviter les déplacements. Troisièmement, selon $\S 7$ (2), les offres de mobilités sont liées aux principes du développement urbain durable, ce qui ajoute une vision normative du développement local et un imaginaire

\footnotetext{
12 «Les modes de transports écologiques (Umweltverbund) doivent donc être prioritaires par rapport au transport privé motorisé en termes d'expansion et de financement de la région métropolitaine (Stadt-Umland-Bereich)» (souligné par moi).

13 «Afin de garantir une offre de transport fiable et ponctuelle, ainsi que pour la réalisation des temps de déplacement attractifs, les transports publics (...) ont la priorité sur les transports privés motorisés dans le cadre du droit applicable. (§26, 5 , souligné par moi).

14 «A l'intérieur du réseau cyclable de Berlin, il convient de définir les liaisons particulièrement importantes pour le cyclisme, en particulier les liaisons d'importance urbaine (réseau prioritaire). Dans le cas des routes désignées comme réseau prioritaire, le cyclisme (...) devrait avoir la priorité sur la circulation individuelle motorisée dans le cadre du droit applicable » (§42, 1, (souligné par moi).
} 
géographique spécifique de la ville. Quatrièmement, une certaine organisation spatiale est vue comme désirable : selon $\$ 7(2,1)$, l’organisation polycentrique est à maintenir et développer. Cinquièmement, l'accessibilité des quartiers est clé $(\$ 7,2,2)$ et de nouveaux développements urbains sont à penser avec les transports publics. Enfin, la motilité étant inégale selon les quartiers berlinois, la loi vise aussi à diminuer les inégalités d'accès aux transports publics. Notamment les «possibilités de mobilité équivalentes dans toutes les parties de Berlin » (§1, souligné par moi) et le fait de « disposer dans tous les quartiers de Berlin d'une offre de transports publics équivalente » $(\S 4,1)$ sont visés.

Système technique. La loi ne s'exprime pas directement sur les systèmes techniques - cette régulation est effectuée à l'échelle de l'UE (directive sur les émissions de la flotte automobile ; directive sur les $\mathrm{NO}_{x}$ ) et la République d'Allemagne (Luftreinhaltungsgesetz) - mais elle précise des éléments techniques et les régulations à travers l'accès à des données publiques partageables concernant la mobilité.

Statut légal. La loi ne s'exprime pas sur le statut légal des personnes, mais reconnaît la mobilité des touristes et ne se réfère donc pas uniquement aux résidents dans la régulation. De même que citoyen ou non n'est pas le problème, mais l'inégalité d'accès à la mobilité en fonction de l'origine géographique des personnes (Herkunft) est thématisée (Préambule).

Corps. La loi s'exprime à la fois sur l'intégrité physique et sur les handicaps physiques des personnes : «Lors de la planification et conception des services et infrastructures de transport, le sentiment de sécurité des personnes est à prendre en compte et leur sécurité personnelle améliorée. Les besoins particuliers de personnes à mobilité réduite des contraintes de mobilités sont à prendre en compte » $(\$ 11)$. Les termes clé sont « sentiment de sécurité » et « contraintes de mobilité »au sens de handicaps physiques, mais aussi la diminution des accidents dans le sens d'une politique « zéro accidents » telle qu'elle existe aujourd'hui dans les villes européennes $(\S 10,3)$.

\subsection{Discussions}


Quatre interprétations peuvent être proposées par rapport à l'hypothèse d'un changement des normes d'habiter depuis 20 ans. Premièrement, on saisit clairement un empowerment d'autres formes de mobilité au détriment de l'automobilité, puisque la priorité ne concerne pas seulement le transport public, mais aussi les piétons et les cyclistes. Elle s'aperçoit aussi dans la valorisation de la rue comme espace public : d'éventuels réaménagements doivent viser à transformer les rues en lieu de rencontre plutôt que de les garder comme lieux de circulation automobile. Enfin, le renforcement de l'accès aux transports publics dans des quartiers qui en sont dépourvus est également l'un des objectifs. Le contexte est celui de la « ville durable » comme modèle d'urbanité où les modes de déplacement écologiques ont la priorité, et où le changement climatique constitue l'horizon prospectif. Ceci peut être lu à travers la montée en puissance des formes de déplacement dont 2/3 à Berlin relèvent de modes alternatifs à l'automobile.

Deuxièmement, on saisit un « mobility turn » de la loi. Il s'agit d'une loi sur la mobilité et non pas sur les transports en ce sens que l'ensemble du problème social des mobilités est appréhendé, et non seulement un secteur des transports. En effet, la mobilité comme pratiques, potentiels, significations, pouvoirs, valeurs (Kaufmann, 2001 ; Lussault \& Stock, 2003 ; Cresswell, 2006) est appréhendée. Cette mobilité n'est pas, pour la loi berlinoise, une fin en soi, mais un moyen pour atteindre un objectif, la «participation à la vie sociale ». Les éléments suivants étayent ce point : les inégalités spatiales (des quartiers) et corporelles (handicaps), les accessibilités des handicapés sont prises en compte ; les multiples mobilités (quotidiennes, touristiques, événementielles, etc.) et les différents moyens de transports sont pris en compte ; les conséquences de la mobilité sur les espaces urbains sont prises en compte; accessibilité et vitesses de déplacement, inter- et multimodalité ; les espaces urbains, climat, rues, sécurité dans l'espace public ; la mobilité ne concerne pas seulement les personnes, mais aussi les biens. Ainsi, un système des mobilités est esquissé et régulé.

Troisièmement, il s'agit bien d'un ordre juridique local en ce sens que la loi est contextuelle et adapté au contexte spatial berlinois : il s'agit d'une capitale $(\S 13)$ où il y a régulièrement des manifestations de masse $(\S 12)$ et une interconnexion à d'autres métropoles $(\S 15)$, la ville se trouve dans l'espace métropolitain Berlin-Brandenburg (§14), la question de la sécurité est posée avec acuité $(\$ \$ 10-11)$ en raison de la lutte quotidienne pour l'espace entre piétons, cyclistes, 
automobilistes, problème récurrent évoqué par les politiques urbaines et la presse. La gestion des conflits d'usage ( $\$ 20-25)$ est ainsi une des questions centrales.

Enfin, la géographicité du droit se lit aussi à travers les catégories spatiales mobilisées dans la loi. D'abord, celle-ci raisonne en termes de «centres » et «quartiers » $(\$ 7,2,2)$ ainsi qu'elle fait référence à la région métropolitaine Berlin-Brandenburg $(\$ 7,1 ; \S 14)$. Ensuite, elle consacre la structure poly-centrique de Berlin à sauvegarder et à développer et évoque les notions « espace public » comme élément central. Le terme « lieu » permet d'exprimer le projet de transformation de la rue comme espace de passage et de flux en un « lieu de rencontre » $(\$ 4,5)$. Enfin, elle évoque Berlin comme lieu de qualité spécifique avec des «fonctions» spécifiques et comme « localisation » $($ Standort $)(\S 13)$ : capitale, mais aussi avec des centralités nationales et mondiales pour le tourisme, la culture, l'enseignement supérieur et la recherche scientifique, les salons et congrès etc. La loi devient ainsi un texte géographique de type normatif en ce sens que la théorie géographique des fonctions urbaines se trouve doublée par un dispositif juridique. La théorie géographique n'est donc plus seulement d'ordre autoréférentiel, mais déploie son efficace dans le monde social à travers un texte juridique's .

De quoi la loi ne parle pas ? Sans pouvoir détailler, on peut mentionner trois éléments. D'abord, la régulation de la mobilité se fait aussi par la vitesse de déplacement : les zones 30 comme moyen de régulation, bien que très utilisé par les Bezirke, ne sont pas évoquées. Ensuite, la zone d'exclusion d'automobiles les plus polluants (Umweltzone) n'est guère évoquée. Enfin, le sharing (à Berlin il y a en 2017 d'environ 3000 voitures en autopartage) n'est problématisé qu'à travers le partage des données, mais non à travers la régulation de son utilisation ${ }^{16}$.

\section{Conclusion}

\footnotetext{
15 Cet aspect pourrait être davantage développé dans la recherche géographique. En Allemagne, notamment la théorie des lieux centraux, au départ un modèle pour analyser la structure et la dynamique spatiale du peuplement, élaboré par Christaller (1933) est devenue un discours normatif par l'insertion dans les plans d'aménagement, et ce à tous les niveaux d'échelles.

16 Depuis 2017 existe une loi fédérale concernant le car sharing $(\mathrm{CsgG})$ qui vise à favoriser l'usage partagé des voitures.
} 
Au-delà de l'étude de cas, trois éléments peuvent être retenus qui permettraient de féconder d'autres études empiriques et d'aider à imaginer de nouveaux cadres interprétatifs. D'abord, la notion « régime de mobilité » permet de focaliser sur la régulation multi-scalaire et multidomaniale de la mobilité au-delà du droit international appréhendé jusqu'ici dans la littérature scientifique. Cette approche permet une articulation entre différentes formes de régulation des mobilités et permet de comprendre les stratégies individuelles qui jouent avec ces multiples normes juridiques. Droit du consommateur (voyage), droit international (tourisme, migration), droit à la libre circulation (UE, Etats-nations), loi sur les secteurs de transports, droit sur les systèmes techniques, etc. définissent les conditions d'accessibilité des individus. Ainsi posé, le régime de mobilité peut être interprété comme étant l'un des éléments du «champs des possibles » ou « motilité » (Kaufmann, 2015), mais non à l'échelle individuelle comme disposition plus ou moins favorable aux déplacements, mais dans sa dimension réglementaire.

Deuxièmement, l'approche par le droit permet d'enrichir la théorie géographique. Appréhender les mobilités comme une pratique fondée sur des droits et bases légales permet une précision supplémentaire dans l'analyse des changements de la motilité et des modes d'habiter mobiles au cours du temps. Elle concernerait aussi la théorie urbaine, car la ville n'est pas seulement un concept géographique, mais aussi un concept juridique (Caillosse, 1995 ; Frug, 1982). Par ailleurs, la notion de « géographicité du droit » permet de saisir les référents spatiaux et catégories spatiales des normes juridiques, les cultures et ordres juridiques à différents niveaux d'échelles ainsi que la régulation des spatialités par les normes juridiques.

Troisièmement, le «mobility turn » de la loi semble un élément intéressant : les législateurs conçoivent dorénavant des lois sur la mobilité en lieu et en place des lois sur le transport. Le terme « mobility turn » est normalement utilisé pour désigner le passage, en sciences sociales, des études sur les modes d'habiter sédentaires vers des modes d'habiter mobiles (cf. Ortar et al., 2018). On peut le mobiliser pour étudier les lois elles-mêmes, l'exemple de Berlin n'en étant qu'une illustration parmi d'autres. Un exemple spectaculaire se trouve dans la tentative récente de l'Etat français de se doter d'une nouvelle loi d'orientation sur la mobilité (LOMI) du 26 décembre 2019 qui remplace la loi d'orientation sur les transports (LOTI) de 1982, qui traduit bien ce tournant mobilitaire jusque dans le titre et les différents articles de la loi. Ainsi, l'article 1 remplace 
systématiquement le terme «transports » de la LOTI par le terme «mobilité » dans la LOMI : « droit au transport » sont remplacés par les mots: «droit à la mobilité », les mots: « système des transports » sont remplacés par les mots : « système des mobilités »; « un service de transport » sont remplacés par les mots: «une solution de mobilité »; « transports public urbain » sont remplacés par les mots : « mobilité », les mots: « modes de déplacement terrestres non motorisés » sont remplacés par les mots: «mobilités actives », etc. ${ }^{17}$.

Un élément non discuté ici, mais qui a un écho important en géographie depuis quelques années, concerne la question du droit à la mobilité et du droit à l'immobilité, analogue à la question d'Henri Lefebvre (1968) sur le droit à la ville et à la centralité. Elle est importante, car elle pose la question du pouvoir et de l'empowerment dans la métropole contemporaine. Qui habite la ville pour quels projets et avec quel statut juridique ? Pour Lefebvre (1968), la réappropriation de la ville par les habitants et la maîtrise du développement urbain étaient l'élément clé du droit à la ville. Force est de constater que les centres des métropoles sont surtout habités de façon temporaire par des personnes mobiles et relativement moins pour y résider. Le pouvoir d'accéder aux centres des villes en tant que résident a donc diminué, celui de l'habiter en tant que consommateur a augmenté. Le « régime de mobilités » semble favoriser un droit temporaire à la ville. Au bout du compte, on peut se poser la question suivante : comment le changement des normes et des manières d'habiter des métropolitains est lié à une régulation de la mobilité qui distribue autrement le droit à la ville et le droit à la mobilité ?

Mathis Stock

Institut de Géographie et Durabilité

Site de Sion - Chemin de l'Institut 18 - Case Postale 4176 - 1950 Sion 4

e-mail : mathis.stock@unil.ch

\section{Bibliographie}

Ascher, F. (2004), « Les sens du mouvement : modernités et mobilités », in Allemand S., Ascher F. et Lévy J. (dir.), Les sens du mouvement, Paris, Belin, 21-34.

17 Cf. la loi dans sa dernière version :

(https://www.legifrance.gouv.fr/affichLoiPreparation.do?idDocument=JORFDOLE000037646678\&type=gene $\underline{\text { ral\&typeLoi }=\text { proj\&legislature }=15 \text { ) }}$ 
Beaude B. (2012), Internet. Changer l'espace, changer la société, Limoges, FYP.

Benda-Beckmann F., Benda-Beckmann K. \& Griffiths A. (2009), Spatializing law. An Anthropological Geography of Law in Society, Farnham, Ashgate.

Blomley, N. (1994), Law, Space, and the Geographies of Power. New York, Guildford.

Blomley, N., Delaney D., Fort R. (éd.) (2001), The legal geographies reader. Law, power, and space. Londres, Blackwell.

Boullier D. (2016), Sociologie du numérique. Paris, Armand Colin.

Bourdieu P. (1990), « Droit et passe-droit». Actes de la recherche en sciences sociales. Vol. 8182, pp. 86-96. DOI : https://doi.org/10.3406/arss.1990.2928

Bourdin A., Lefeuvre M.-F., Melé P. (2006), Les règles du jeu urbain. Entre droit et confiance. Paris, Descartes et Cie.

Braverman, I., Blomley N., Delaney, D. \& Kedar, A. (éd.) (2014), The Expanding Spaces of Law: A Timely Legal Geography. Stanford, Stanford University Press.

Brighenti, A. (2006), « On Territory as Relationship and Law as Territory », Canadian Journal of Law and Society, 21(2), 65-86.

Caillosse J. (1995), « La ville, le droit et la redistribution des territoires administratifs », Politique et management public, $\mathrm{n}^{\circ} 3$, p. 83-119

Christaller W. (1980), Die zentralen Orte in Süddeutschland. Eine ökonomisch-geographische Untersuchung über die Gesetzmäßigkeit der Verbreitung und Entwicklung der Siedlungen mit städtischen Funktionen, Darmstadt, Wissenschaftliche Buchgesellschaft (1 ère éd. 1933).

Cresswell, T. (2006), On the Move. Mobility in the Modern Western World, Londres, Routledge.

Cresswell, T. (2010), « Towards a Politics of Mobility », Environment and Planning Part D:

Society and Space, $\mathrm{n}^{\circ} 1$, p. 17-31.

Delaney, D. (2003), Law and nature. Cambridge, Cambridge University Press.

Delaney, D., Blomley, N. \& Ford, R. (2001), « Where is law? », In: Blomley, N., Delaney D. \&

Ford F. (Hg.) (2001), The Legal Geographies Reader: Law, power, and space. London:

Blackwell, pp xiii-xxii.

Delaney D. (2015), « Legal geography I: Constitutivities, complexities, and contingencies ». Progress in Human Geography, 39(1), 96-102.

Delaney D. (2016), « Legal geography II: Discerning Injustice ». Progress in Human Geography, 40(1), 1-8.

Delaney D. (2017), « Legal geography III: New worlds, new convergences ». Progress in Human Geography, 41(5), 667-675

Delmas-Marty, M. (2004), Le relatif et l'universel. Les forces imaginantes du droit 1. Paris, Seuil.

Erbsen, A. (2011), « Constitutional Spaces », Minnesota Law Review, 95(X), 1168-1267

Ford, R. (1994), « The Boundaries of Race: Political Geography in Legal Analysis », Harvard Law Review, 107(8), 1841-1921

Ford, R. (1999), « Law's Territory (A History of Jurisdiction) », Michigan Law Review, 97(4), 843-930

Forest, P. (dir.) (2009), Géographie du droit. Epistémologie, développement et perspectives, Québec, Presses de l'Université de Laval. 
Frug, G. (1980), « The City as a Legal Concept », Harvard Law Review, 93, 1057-1154 Garcier, R. (2009), « Le Droit et la fabrique de l'espace. Aperçus méthodologiques sur l'usage des sources juridiques en géographie », In Forest P. (dir.), Géographie du droit. Épistémologie, développement et perspectives. Québec, Presses universitaires de Laval, p. 69-90.

Garcier, R. (2014), « Disperser, confiner ou recycler ? Droit, modes de gestion et circulations spatiales des déchets faiblement radioactifs en France ", L'Espace géographique, vol. 43, pp. 265-283

Glick Schiller N. \& Salazar N. (2013), « Regimes of mobility across the globe ». Journal of Ethnic and Migration, 39 (2), 183-200.

Gössling S. (2019), Celebrities, air travel, and social norms, Annals of Tourism Research, vol.79, 102775 (https://doi.org/10.1016/j.annals.2019.102775)

Gregory, D. (1994) Geographical Imaginations. Oxford : Blackwell

Hannam, K., Sheller, M. et Urry, J. (2006), « Editorial. Mobilities, Immobilities and Moorings », Mobilities 1, p. 1-22.

Harvey, D. (1989), The Condition of Postmodernity. An Enquiry into the Origins of Cultural Change. Oxford, Blackwell.

Kaufmann, V. (2013), « Mobilité », in : J. Lévy et M. Lussault (dir.), Dictionnaire de la géographie et de l'espace des sociétés, Paris, Belin, 677-679.

Koslowski R. (2011), « Global Mobility Regimes: A Conceptual Framework », dans Koslowski R. (éd.), Global Mobility Regimes, New York, Palgrave Macmillan, p. 1-25.

Lefebvre H. (1968), Le droit à la ville. Paris, Anthropos.

Lévy J. (2013), « Géographicité », in : J. Lévy et M. Lussault (dir.), Dictionnaire de la géographie et de l'espace des sociétés, Paris, Belin, 433-436

Lévy, J. (1994), L'espace légitime. Sur la dimension géographique de la fonction politique. Paris, Presses de la Fondation Nationale des Sciences Politiques.

Lévy, J., Fauchille, J.-N., \& Póvoas, A. (2018), Théorie de la justice spatiale. Paris: Odile Jacob. Lussault, M. (2007) L’homme spatial. Paris, Seuil.

Lussault, M. \& Stock M. (2003), « Mobilité », in : J. Lévy et M. Lussault (dir.), Dictionnaire de la géographie et de l'espace des sociétés, Paris, Belin, 622-625.

Maccaglia F. (2009), Palerme, illégalisme et gouvernement urbain d'exception. Sociétés, espaces, temps. Lyon, ENS Éditions.

Mélé, P. (2009), « Pour une géographie du droit en action », Géographie et cultures, n 72, p.2543.

Ortar N., Salzbrunn M., Stock M. (2018), Migrations, circulations, mobilités. Nouveaux enjeux épistémologiques à l'épreuve du terrain, Aix, Presses Universitaires de Provence.

Philippopoulos-Mihalopoulos, A. (2011), « Law’s Spatial Turn: Law, Justice and a Certain Fear of Space », Law, Culture and the Humanities, 7(2), 187-202

Pirie, G. H. (1983), « On Spatial Justice », Environment and Planning A 15, 465-73.

PRIES L. (2008), Die Transnationalisierung der sozialen Welt: Sozialräume jenseits von Nationalgesellschaften, Frankfurt am Main, Suhrkamp.

Prytherch D. (2012), « Legal Geographies - Codifying the Right-Of-Way: Statutory Geographies 
of Urban Mobility and The Street », Urban Geography, 33:2, 295-314, DOI: 10.2747/0272-3638.33.2.295

Raffestin C. (1989), « Théorie du réel et géographicité », EspacesTemps, 40-41, 26-31

Retaillé D. (2003), « Géographicité », J. Lévy et M. Lussault (dir.), Dictionnaire de la géographie et de l'espace des sociétés, Paris, Belin, 398-399.

Senat Berlin (2018), Konzept für einen stadtverträglichen und nachhaltigen Berlin-Tourismus 2018+ [online]. Berlin: Senat Berlin. Repéré à:

https://www.berlin.de/sen/wirtschaft/wirtschaft/branchen/tourismus/tourismuskonzept2018.pdf [accédé le 7 février 2020].

Senat Berlin (2017), Mobilität der Stadt. Berliner Verkehr in Zahlen [online]. Berlin: Senat Berlin. Repéré à :

https://www.berlin.de/senuvk/verkehr/politik planung/zahlen fakten/download/Mobilitaet dt ko mplett.pdf [accédé le 7 février 2020].

Shamir R. (2005), « Without Borders? Notes on Globalization as a Mobility Regime », Sociological Theory, vol.23, n², 197-217

Sheller, M. \& Urry, J. (2006), « The New Mobilities Paradigm », Environment and Planning A 2, 207-226.

Siehr, A. (2016), Das Recht am öffentlichen Raum. Theorie des öffentlichen Raumes und die räumliche Dimension von Freiheit, Tübingen, Mohr Siebeck.

Soja, E. (2010), Seeking spatial justice, Minnesota, University of Minnesota Press.

Stock, M. (2015), « Habiter comme « faire avec l'espace ». Réflexions à partir des théories de la pratique », Annales de Géographie, n704, 421-441.

Stock, M. (2006), « L'hypothèse de l'habiter poly-topique : pratiquer les lieux géographiques dans les sociétés à individus mobiles », Espacestemps.net, Textuel, 26.02.2006 (http://www.espacestemps.net/en/articles/lrsquohypothese-de-lrsquohabiter-poly-topiquepratiquer-les-lieux-geographiques-dans-les-societes-a-individus-mobiles-en/)

Stock, M. (2003-2004), « Pratiques des lieux, styles d'habiter, régimes d'habiter. Pour une analyse trialogique des dimensions spatiales des sociétés humaines ", Travaux de l'Institut de Géographie de Reims, vol. 115-118, pp. 213-229, DOI : https://doi.org/10.3406/tigr.2003.1473 Thrift, N. (1996), Spatial Formations. London, Sage.

Valverde, M. (2005), « Taking 'land use' seriously: toward an ontology of municipal law », Law/Text/Culture, 9, 34-59.

Werlen, B. (1995), Sozialgeographie alltäglicher Regionalisierung, Vol. 1:Ontologie von Raum und Gesellschaft, Stuttgart, Steiner.

Werlen, B. (1996), « Geographie globalisierter Lebenswelten », Österreichische Zeitschrift für Soziologie, 21(2), 97-128.

Werlen, B. (1995), Sozialgeographie alltäglicher Regionalisierung, Vol. 2: Globalisierung, Region und Regionalisierung, Stuttgart, Steiner.

Zelinsky, W. (1971), The Hypothesis of the Mobility Transition. Geographical Review, 61/2, 219-249. 
PRE-PRINT : ANNALES DE GEOGRAPHIE - 2020

Zick, T. (2006), Speech and spatial tactics, Texas Law Review, 84(3), 581-651. 